Check for updates

Cite this: Green Chem., 2020, 22 565

DOI: $10.1039 / c 9 g c 90121 c$

rsc.li/greenchem

\section{Correction: Efficient separation of immiscible oil/water mixtures using a perforated lotus leaf}

Chunhui Zhang, (DD a,b Yuheng Zhang, (D) ${ }^{c}$ Xiao Xiao, (D) ${ }^{c}$ Guoliang Liu, (D) ${ }^{c}$ Zhe Xu, (D) ${ }^{c}$ Bing Wang, (iD a Cunming Yu, (D) *c Robin H. A. Ras (D) ${ }^{\text {d,e }}$ and Lei Jiang (iD) a,c

Correction for 'Efficient separation of immiscible oil/water mixtures using a perforated lotus leaf' by Chunhui Zhang et al., Green Chem., 2019, 21, 6579-6584.

The authors regret that Fig. 2 and 3 are incorrect. The correct figures are as follows:

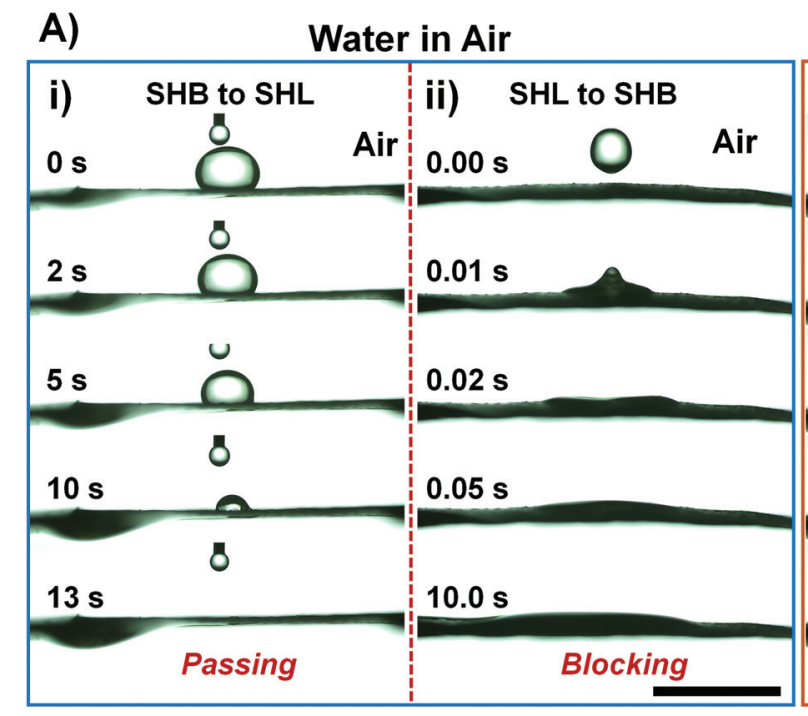

B) Oil in Water

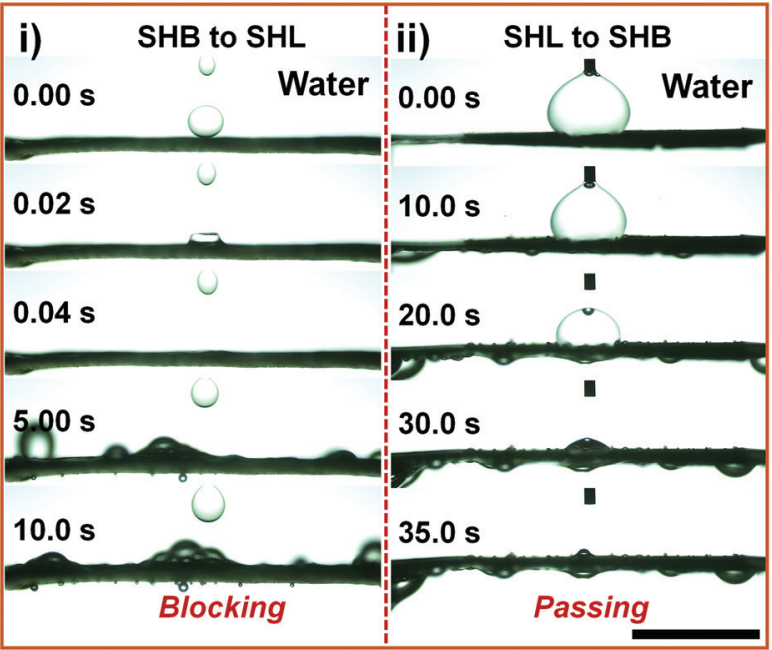

C)

D)
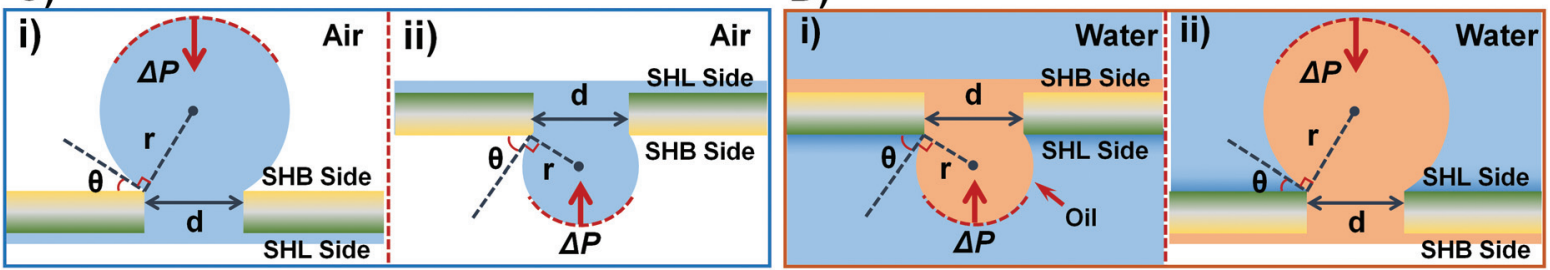

Fig. 2 Unidirectional permeation processes of water/oil droplets and their underlying mechanisms. (A) Unidirectional permeation of water in air. The water droplet can spontaneously pass through the perforated lotus leaf from the SHB side to the SHL side. But the water droplet cannot pass through the perforated lotus leaf in the opposite direction. (B) In contrast, the oil droplet cannot pass through the perforated lotus leaf from the SHB side to the SHL side whereas it successfully permeates from the SHL side to the SHB side. (C and D) Illustration of the mechanism for the unidirectional permeation of water and oil, which is mainly attributed to the asymmetric Young-Laplace pressure. Scale bar: $1 \mathrm{~cm}$.

\footnotetext{
${ }^{a}$ Laboratory of Bio-Inspired Materials and Interface Sciences, Technical Institute of Physics and Chemistry, Chinese Academy of Sciences, Beijing 100190, China ${ }^{b}$ School of Chemical Sciences, University of Chinese Academy of Sciences, Beijing 100049, China

${ }^{c}$ Key Laboratory of Bio-Inspired Smart Interfacial Science and Technology of Ministry of Education, School of Chemistry, Beijing Advanced Innovation Centre for Biomedical Engineering, Beihang University, Beijing 100191, China. E-mail: ycmbhs@iccas.ac.cn

${ }^{d}$ Department of Applied Physics, Aalto University School of Science, Puumiehenkuja 2, 02150 Espoo, Finland

${ }^{e}$ Department of Bioproducts and Biosystems, Aalto University School of Chemical Engineering, Kemistintie 1, 02150 Espoo, Finland
} 

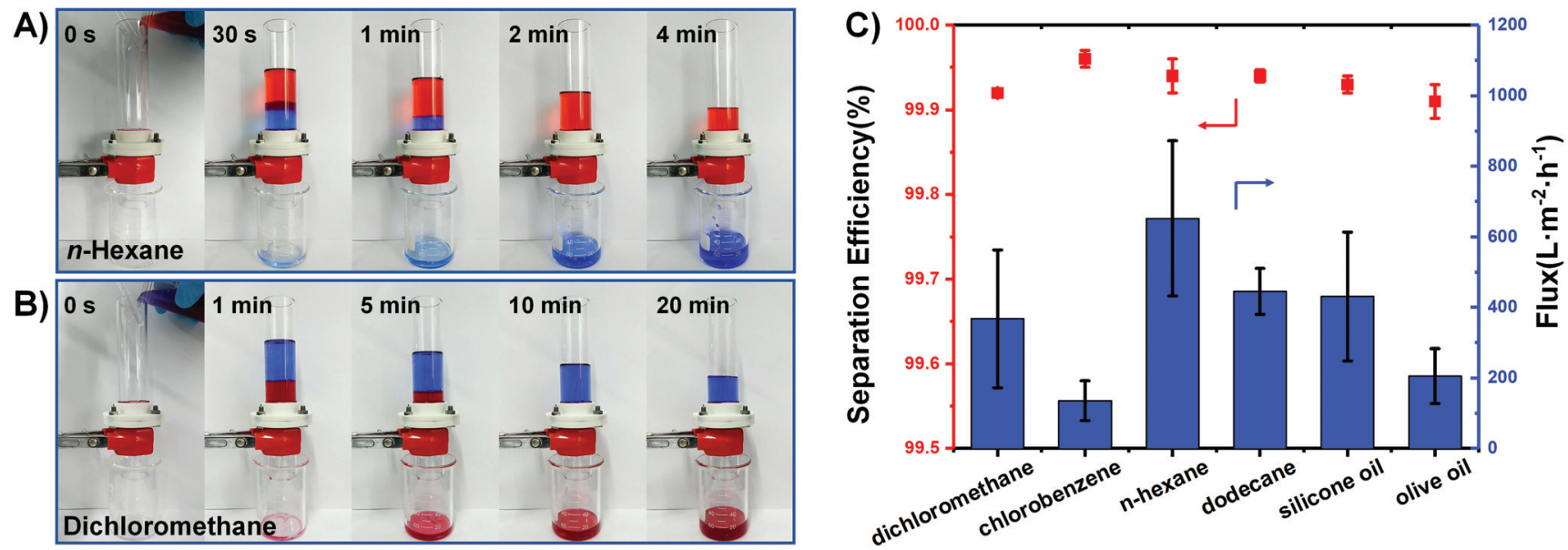

Fig. 3 Separation of the mixture of oil (red colour) and water through the perforated lotus leaf. (A) The separation processes of the mixture of light oil ( $n$-hexane) and water. (B) The separation processes of the mixture of heavy oil (dichloromethane) and water. (C) Separation efficiency and flux of the perforated lotus leaf versus different oil/water mixtures.

The Royal Society of Chemistry apologises for these errors and any consequent inconvenience to authors and readers. 